\title{
PERFORMANCE ANALYSIS OF MISO-OFDM SYSTEM WITH DELAYED FEEDBACK
}

\author{
Suraj Gawande ${ }^{1}$, K. Vinoth Babu ${ }^{2}$, G. Ramachandra Reddy ${ }^{3}$, Bibin Baby John ${ }^{4}$ \\ ${ }^{1}$ Student, School of Electronics Engineering, VIT University, Tamilnadu, India \\ ${ }^{2}$ Assistant Professor (Senior), School of Electronics Engineering, VIT University, Tamilnadu, India \\ ${ }^{3}$ Dean and Professor (Senior), School of Electronics Engineering, VIT University, Tamilnadu, India \\ ${ }^{4}$ Student, School of Electronics Engineering, VIT University, Tamilnadu, India
}

\begin{abstract}
Orthogonal Frequency Division Multiplexing (OFDM) is combined with Multiple Input Multiple Output (MIMO) systems in recent wireless standards to provide high data rate with reliable communication. Improvement in performance of the system can be achieved when Channel State Information (CSI) is used at the transmitter to perform adaptation. The diversity gains are diminished when the adaptations are done with the delayed outdated CSI. Here we derive the closed-form average Bit Error Rate (BER) expression for Multiple Input Single Output (MISO)-OFDM system, as a function of correlation $\left({ }^{\rho}\right)$ between perfect CSI and delayed CSI. Here, we develop an analytical model which explains the effect of feedback delay. The simulation results for analytical model also prove that the diversity gain of considered MISO-OFDM system decreases for $\rho<1$.
\end{abstract}

Keywords: MISO, OFDM, CSI, Average BER, Average Spectral efficiency.

\section{INTRODUCTION}

The key challenge faced by next generation wireless communication system is to provide high data rate with high Quality of Service (QoS). OFDM provides high speed data transmission in frequency selective channels which in turn enhances spectral efficiency. MIMO is another advanced physical layering technique which provides spatial diversity gains to mitigate the effects of fading. Hence to meet the mounting demands of high data rate with reliable communication, recent wireless standards combine OFDM and MIMO systems. MIMO-OFDM is used in wireless standards such as Long Term Evolution (LTE), Long Term Evolution-Advanced (LTE-A), IEEE 802.16, IEEE 802.11, etc. to achieve better QoS [1].

In closed loop MIMO system, channel is estimated at the receiver side and the CSI is fed back to the transmitter side which is also termed as Channel State Information at the transmitter side (CSIT). Through literature studies it is known that having CSI at transmitter side, leads to improvement in the efficiency of data transmission by updating the transmission parameters according to channel quality. Therefore, we can improve the performance of the wireless communication systems by performing adaptation based on the perfect CSI at the transmitter side [2,3] i.e. If the CSI is known at the transmitter side then we can go for any of the techniques like precoding or transmit beamforming [4], adaptive bit loading, adaptive power loading and variable code rate, to improve the overall performance of the system. Therefore, it is very important to have perfect CSIT.

In Frequency Division Duplex (FDD) system, channel estimation is performed at the receiver and via a dedicated feedback channel, CSI is fed back to the transmitter. But in practice, the feedback channel is band limited and in MIMOOFDM systems, with the increase in number of subcarriers and number of antennas, the amount of CSI data also grows linearly. Therefore, when the data transmitted through the limited feedback channel increases, it leads to occurrence of non-zero feedback delay $[5,6]$.

The present wireless standards offers high data rate even at high mobility. LTE systems are expected to offer reliable services even at $350 \mathrm{kmph}$ mobility. While LTE-A are expected to offer reliable services even at $500 \mathrm{kmph}$ mobility [7]. High mobility leads to reduction in coherence time which makes the channel time varying [8].

The non-zero delay and time varying nature of channel makes CSIT outdated with respect to the actual channel. Thus the adaptations that need to be carried out based on the CSIT become futile because of the outdated CSIT, which in turn degrades the performance of the MIMO-OFDM system. Thus the non-zero feedback delay diminishes the diversity gains offered by MIMO-OFDM system [9].

In this paper, we have developed an analytical model which states the effects of non-zero feedback delay. The analytical 
model is also validated with the simulation results. The rest of the paper is organized as follows: Section 2 describes the analytical model. Section 3 presents simulation results and Section 4 concludes the paper.

\section{ANAL YTICAL MODEL}

The block diagram of considered MISO-OFDM system is shown in Fig.1. Here the modulated symbols are encoded through SFBC encoder [10]. The encoded symbols are passed through IFFT blocks which generate OFDM symbols. These OFDM signals are transmitted through independent identical distribution (i.i.d) frequency selective multipath fading channel. At the receiver section, the received signal is passed through FFT block, and then SFBC decoding process is carried out to obtain detected symbols. The channel is estimated at receiver section (CSIR) which is fed back through feedback channel to transmitter side (CSIT).

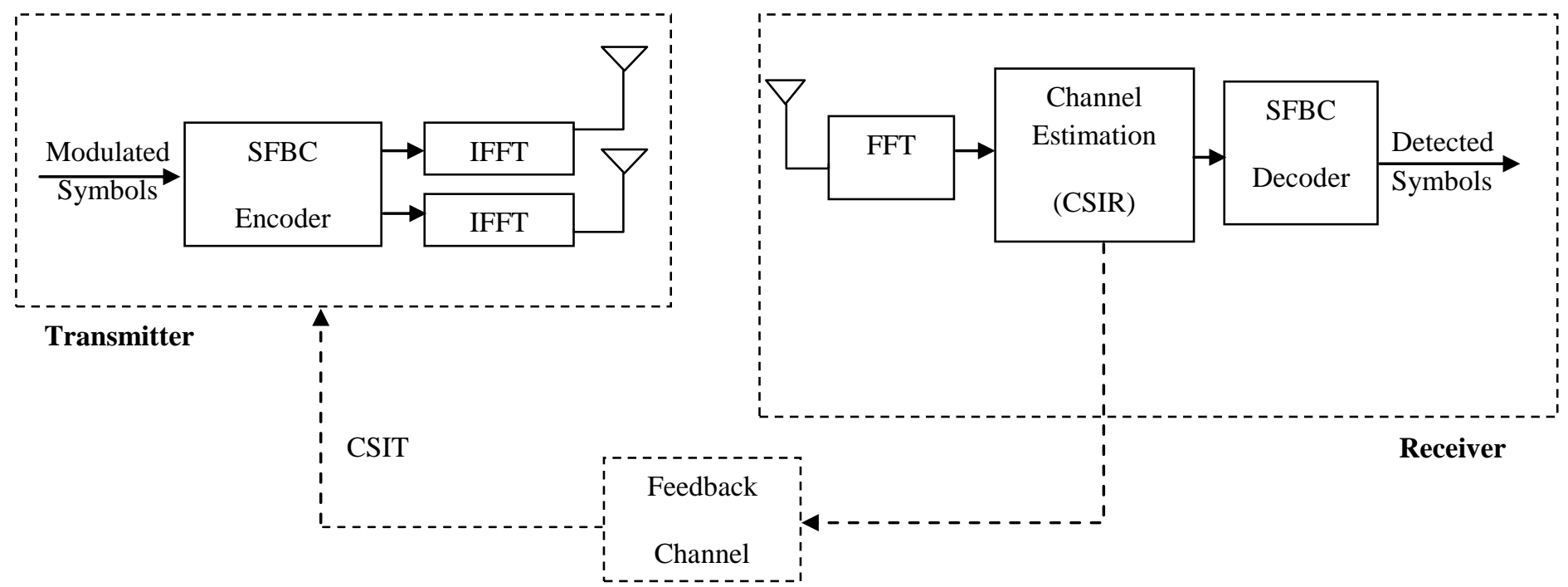

Fig.1 Block diagram of the closed loop MISO-OFDM system.

\subsection{Average BER Expression for M-Quadrature Amplitude Modulation (QAM)-MISO-OFDM System}

Now we derive the closed-form average BER expression for MISO-OFDM system.

Let us represent the correlation function between actual channel frequency response $\mathrm{H}_{\mathrm{I}}(\mathrm{m})$ and the delayed outdated estimate of channel frequency response $\mathrm{H}_{\mathrm{I}}(\mathrm{m})$ as $\rho$.

where $0 \leq \rho \leq 1$.

In general

$\mathrm{E}[\mathbf{H} \mathbf{H}]=\rho \mathbf{I}$

where $\mathbf{H}=[\mathrm{H}(0), \mathrm{H}(1), \ldots \mathrm{H}(\mathrm{L}-1)]^{\mathrm{T}}$. I is the identity matrix of size L X L. For simplicity, symbol and subcarrier indexes are ignored in the above expression.

It is easy to determine probability density function (pdf) of $|\hat{\mathrm{H}}|^{2}$ but it is very difficult to determine the pdf of $|\mathrm{H}|^{2}$.To resolve this issue, we first determine the pdf of $\mid \mathrm{H}^{2}$ conditioned on $|\mathrm{H}|^{2}$ To model this we use Gauss Markov model where actual and delayed CQI is related by [11]

$$
\mathrm{H}=\rho \mathrm{H}+\sqrt{1-\rho^{2}} \delta
$$

where an error term. Let $\delta$ has Complex Normal distribution with zero mean and variance 1 . Therefore for a given $\mathrm{H}$ we can write,

$$
\mathrm{H} \square \mathrm{CN}\left(\rho \mathrm{H}, 1-\rho^{2}\right)
$$

Let us represent $|\mathrm{H}|^{2}$ and $|\mathrm{H}|^{2}$ as $\mathrm{Z}$ and $\mathrm{Z}$ respectively. Also take $\sqrt{\rho^{2} Z_{a s}}$ B'For a given B', the pdf of $Z$ follows non central Chi square distribution with two degrees of freedom. It can be represented as,

$\mathrm{p}_{\mathrm{Z} \mid \mathrm{B}^{\prime}}\left(\mathrm{z} / \mathrm{b}^{\prime}\right)=\frac{\mathrm{e}^{-\left(\mathrm{z}+\mathrm{b}^{\prime^{2}}\right) /\left(1-\rho^{2}\right)}}{1-\rho^{2}} \mathrm{~J}_{0}\left(\frac{2 \mathrm{j} \sqrt{\mathrm{z}} \mathrm{b}^{\prime}}{1-\rho^{2}}\right)$

The pdf of $\hat{Z}$ for MISO system with $\mathrm{N}_{\mathrm{T}}$ transmitting antennas can be derived from order statistics as [12] 


$$
\mathrm{p}_{\mathrm{Z}}(\hat{\mathrm{z}})=\mathrm{N}_{\mathrm{T}} \sum_{\mathrm{i}=0}^{\mathrm{N}_{\mathrm{T}}-1}\left(\begin{array}{c}
\mathrm{N}_{\mathrm{T}}-1 \\
\mathrm{i}
\end{array}\right)(-1)^{\mathrm{i}} \mathrm{e}^{-(\mathrm{i}+1) \hat{\mathrm{z}}}
$$

From the relationship $B^{\prime}=\sqrt{\rho^{2} Z}$ the pdf of $\quad B^{\prime}$ can be given as,

$\mathrm{p}_{\mathrm{B}^{\prime}}\left(\mathrm{b}^{\prime}\right)=\frac{2 \mathrm{~b}^{\prime} \mathrm{N}_{\mathrm{T}}}{\rho^{2}} \sum_{\mathrm{i}=0}^{\mathrm{N}_{\mathrm{T}}-1}\left(\begin{array}{c}\mathrm{N}_{\mathrm{T}}-1 \\ \mathrm{i}\end{array}\right)(-1)^{\mathrm{i}} \mathrm{e}^{-\left((\mathrm{i}+1) \mathrm{b}^{\prime 2} / \rho^{2}\right)}$

$\mathrm{p}_{\mathrm{z}}(\mathrm{z})$ is given as,

$$
\mathrm{p}_{\mathrm{Z}}(\mathrm{z})=\int_{0}^{\infty} \mathrm{P}_{\mathrm{Z} \mid \mathrm{B}^{\prime}}\left(\mathrm{z} / \mathrm{b}^{\prime}\right) \mathrm{P}_{\mathrm{B}}\left(\mathrm{b}^{\prime}\right) \mathrm{db^{ \prime }}
$$

Substituting (4) and (6) in (7) gives,

$$
\begin{aligned}
\mathrm{p}_{\mathrm{Z}}(\mathrm{z}) & =\frac{2 \mathrm{~N}_{\mathrm{T}}}{\rho^{2}\left(1-\rho^{2}\right)} \sum_{\mathrm{i}=0}^{\mathrm{N}_{\mathrm{T}}-1}\left(\begin{array}{c}
\mathrm{N}_{\mathrm{T}}-1 \\
\mathrm{i}
\end{array}\right)(-1)^{\mathrm{i}} \\
& \left\{\left\{\int_{0}^{\infty} \mathrm{b}^{\prime} \mathrm{e}^{-\left(\mathrm{z}+\mathrm{b}^{2}\right) /\left(1-\rho^{2}\right)} \mathrm{e}^{-\left((\mathrm{i}+1) \mathrm{b}^{2} / \rho^{2}\right)} \mathrm{J}_{0}\left(\frac{2 \mathrm{j} \sqrt{\mathrm{z}} \mathrm{b}^{\prime}}{1-\rho^{2}}\right) \mathrm{db}^{\prime}\right\}\right.
\end{aligned}
$$

Considering the integral part in (8)

$$
\begin{aligned}
& \int_{0}^{\infty} b^{\prime} \cdot e^{-\left(z+b^{\prime 2}\right) /\left(1-\rho^{2}\right)} e^{-\left((i+1) b^{\prime 2} / p^{2}\right)} J_{0}\left(\frac{2 j \sqrt{z} b^{\prime}}{1-\rho^{2}}\right) d b^{\prime} \\
& =\int_{0}^{\infty} b^{\prime} \cdot e^{\frac{-\left(\rho^{2}+(i+1)\left(1-\rho^{2}\right)\right) b^{\prime 2}}{\rho^{2}\left(1-\rho^{2}\right)}} J_{0}\left(\frac{2 j \sqrt{z} b^{\prime}}{1-\rho^{2}}\right) d b^{\prime}
\end{aligned}
$$

Considering the standard integral

$$
\int_{0}^{\infty} x^{u} e^{-\alpha x^{2}} J_{v}(\beta x) d x=\frac{\Gamma\left(\frac{1}{2} v+\frac{1}{2} u+\frac{1}{2}\right)}{\beta \alpha^{\frac{1}{2}} \Gamma(v+1)} e^{-\left(\frac{\beta^{2}}{8 \alpha}\right)} \cdot M_{\frac{1}{2} u, \frac{1}{2} v}\left(\frac{\beta^{2}}{4 \alpha}\right)
$$

Comparing (9) with (10) gives,

$$
\begin{aligned}
& u=1 \\
& v=0 \\
& \alpha=\frac{\left(\rho^{2}+(i+1)\left(1-\rho^{2}\right)\right)}{\rho^{2}\left(1-\rho^{2}\right)} \\
& \beta=\frac{2 j \sqrt{z b^{\prime}}}{1-\rho^{2}}
\end{aligned}
$$

Substituting (11) in (10) gives the integral of (9).

$$
=\frac{\left(1-\rho^{2}\right)}{2 j \sqrt{z}} \frac{\rho\left(1-\rho^{2}\right)^{1 / 2}}{\left[\rho^{2}+(i+1)\left(1-\rho^{2}\right)\right]^{1 / 2}} \mathrm{e}^{\frac{z \rho^{2}}{2(1-\rho 2)\left(\rho^{2}+(i+1)\left(1-\rho^{2}\right)\right)}} \mathrm{M}_{\frac{1}{2}, 0}\left(\frac{\frac{-4 z}{\left(1-\rho^{2}\right)^{2}}}{4\left(\frac{\left[\rho^{2}+(i+1)\left(1-\rho^{2}\right)\right]}{\rho^{2}\left(1-\rho^{2}\right)}\right]}\right)
$$

The standard Whittaker function is given by,

$$
\mathbf{M}_{\lambda, \mathrm{u}}(\mathbf{Z})=\mathbf{Z}^{\mathrm{u}+1 / 2} \mathrm{e}^{-\square / 2} \Phi\left(\mathrm{u}-\lambda+\frac{1}{2}, 2 \mathrm{u}+1 ; \mathbf{Z}\right)
$$

Comparing the last term in (12) with (13), we get

$$
\lambda=1 / 2, u=0, \mathbf{Z}=\frac{\frac{-z}{\left(1-\rho^{2}\right)^{2}}}{\left[\frac{\left[\rho^{2}+(i+1)\left(1-\rho^{2}\right)\right]}{\rho^{2}\left(1-\rho^{2}\right)}\right]}
$$

Last term in (13) is a confluent hyper geometric function which is given as,

$\phi(\alpha, \mathrm{y}: \mathbf{Z})=1+\frac{\alpha \mathbf{Z}}{\mathrm{y} !}+\frac{\alpha(\alpha+1) \mathbf{Z}^{2}}{\mathrm{y}(\mathrm{y}+1) \mathbf{Z} !}+\ldots$

Substituting (14) in (13), we get

$$
\mathbf{M}_{(1 / 2,0)}(\mathbf{Z})=\mathbf{Z}^{1 / 2} \mathrm{e}^{-\mathbf{Z} / 2} \phi(0,1 ; \mathbf{Z})
$$

Thus equation (16) reduces to

$$
M_{(1 / 2,0)}(\mathbf{Z})=\mathbf{Z}^{1 / 2} e^{-\mathbf{Z} / 2}
$$

Substituting (17) in (12) and simplifying gives us

$$
\mathrm{P}_{\mathrm{Z}}(\mathrm{Z})=\mathrm{N}_{\mathrm{T}} \sum_{\mathrm{i}=0}^{\mathrm{N}_{\mathrm{T}}-1}\left(\begin{array}{l}
\mathrm{N}_{\mathrm{T}}-1 \\
\mathrm{i}
\end{array}\right) \frac{(-1)^{\mathrm{i}} \mathrm{e}^{-(\mathrm{i}+1) \mathrm{Z} /\left(\mathrm{m}+1-\mathrm{m} \rho^{2}\right)}}{\mathrm{m}+1-\mathrm{m} \rho^{2}}
$$

The instantaneous BER of M-QAM modulation with ' $b$ ' bits/symbol is given

$$
P_{e}=0.2 e^{-1.6 \gamma_{s} z / 2^{b}-1}
$$

Where $\gamma_{s}$ is Average Signal to Noise ratio.

The average BER can be expressed as,

$$
\overline{\mathrm{P}_{\mathrm{e}}}=\int_{\mathrm{z}=0}^{\infty} \mathrm{P}_{\mathrm{e}} \mathrm{P}_{\mathrm{Z}}(\mathrm{z}) \mathrm{dz}
$$

Substituting (18) and (19) in (20) and simplifying gives,

$$
\overline{\mathrm{P}_{\mathrm{e}}}=0.2 \mathrm{~N}_{\mathrm{T}} \sum_{\mathrm{i}=0}^{\mathrm{N}_{\mathrm{T}}-1}\left(\begin{array}{c}
\mathrm{N}_{\mathrm{T}}-1 \\
\mathrm{i}
\end{array}\right) \frac{(-1)^{\mathrm{i}}\left(2^{\mathrm{b}}-1\right)}{1.6 \gamma_{\mathrm{s}}\left(\mathrm{i}+1-\mathrm{i} \rho^{2}\right)+(\mathrm{i}+1)\left(2^{\mathrm{b}}-1\right)}
$$

\subsection{Average BER Expression for M-QAM-Single Input Single Output (SISO)-OFDM SYSTEM}

The pdf of for SISO system can be reduced from (5) as, $\mathrm{p}_{\overline{\mathrm{z}}}(\hat{\mathrm{z}})=\mathrm{e}^{\mathrm{Z}} ; \hat{\mathrm{z}}>0$ 
The pdf of B' for SISO can be given as,

$p_{B^{\prime}}\left(b^{\prime}\right)=\frac{2 b^{\prime}}{\rho^{2}} e^{-\left(b^{\prime 2} / \rho^{2}\right)}$

The pdf of $Z$ can be found by substituting (22) and (23) in (7). Using same steps from (8) to (17) the pdf of $\mathrm{Z}$ can be derived as,

$$
\mathrm{p}_{\mathrm{Z}}(\mathrm{z})=\mathrm{e}^{-\mathrm{z}}
$$

Substituting (24) and (19) in (20), we get average BER for SISO system as,

$$
\overline{P_{e}}=\frac{0.2\left(2^{b}-1\right)}{1.6 \gamma_{s}+2^{b}-1}
$$

\section{RESULTS}

In this section, the performance of the MISO-OFDM system is evaluated by computer simulation for frequency selective fading channel with 10 taps $(\mathrm{N}=10)$. The channel taps are complex Gaussian random processes with zero mean and variance 1 / NThe OFDM system includes 64 subcarriers. Here, we consider two transmitter antennas $\left(N_{T}=2\right)$ and one receiver antenna $\left(N_{R}=1\right)$ with 32 QAM constellation $(\mathrm{M}=32)$.

Fig. 2 shows Average BER against SNR curves for different values of that as $\rho$ decreases, there is degradation in performance of the system.

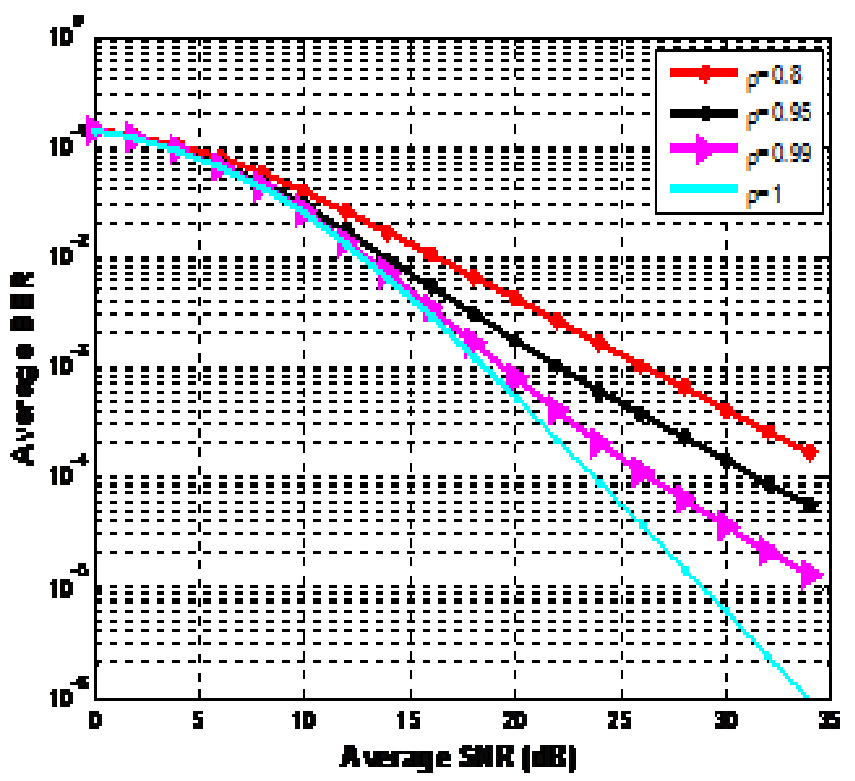

Fig 2 Average BER vs. Average SNR (dB)

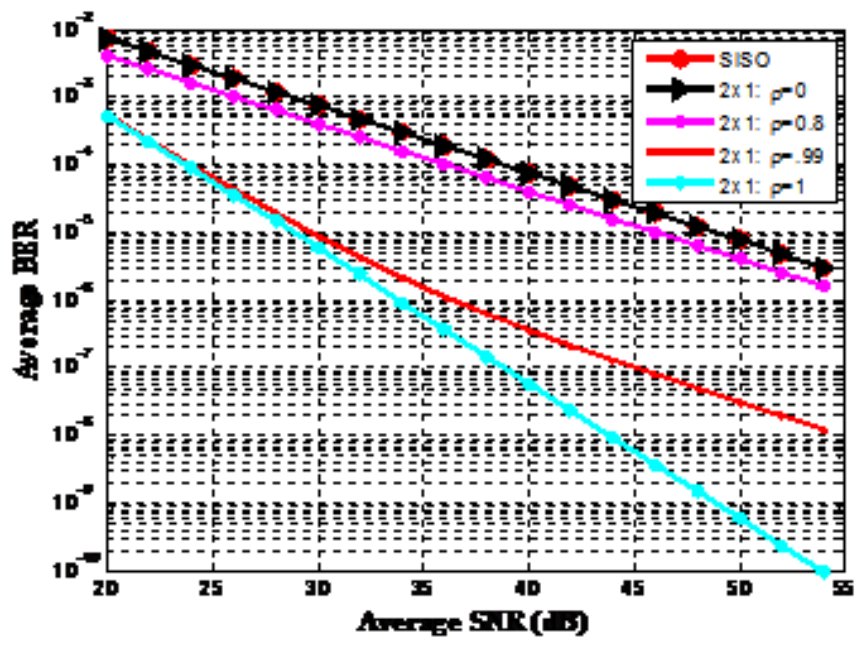

Fig.3. Average BER vs. Average SNR (dB)

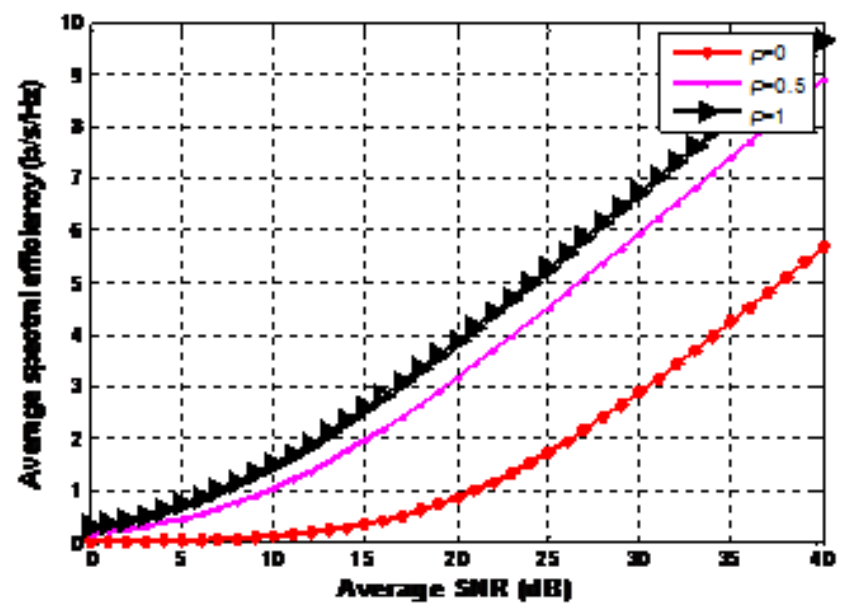

Fig.4. Average Spectral Efficiency (R) vs. Average SNR (dB)

Fig.3 shows Average BER comparison between SISO-OFDM system and for MISO-OFDM system for different values of such as $0, \rho 0.8,0.99$ and 1 . MISO-OFDM system with $\mathrm{N}_{\mathrm{T}}=2$ and $\mathrm{N}_{R}=1$ is denoted as $2 \times 1$. From Fig.3, we can see that BER performance of SISO-OFDM system and MISO-OFDM system with $\rho=0$ is same. This proves that MISO system will act as SISO system when correlation $\rho=0$

From other curves it is clear that diversity gain of MISOOFDM system decreases for values of $\rho<1$.

Fig.4 shows Average spectral efficiency (R) vs. SNR for MISO-OFDM system for different values of $\beta$ uch as $0,0.5$, and 1.The average spectral efficiency $(\mathrm{b} / \mathrm{s} / \mathrm{Hz})$ can be calculated from

$$
\mathrm{R}=\mathrm{E}\left[\frac{1}{\mathrm{~W}\left(\mathrm{~T}_{\mathrm{U}}+\mathrm{T}_{\mathrm{CP}}\right)} \sum_{\mathrm{m}=0}^{\mathrm{L}-1} \mathrm{~b}(\mathrm{~m})\left[1-\overline{\mathrm{P}}_{\mathrm{e}}(\mathrm{m})\right]\right]
$$


where, $\mathrm{W}$ is total system Bandwidth, $\mathrm{T}_{\mathrm{U}}$ is useful symbol duration, $\mathrm{T}_{\mathrm{CP}}$ is cyclic duration, $\mathrm{b}$ is bits loaded on $\mathrm{m}^{\text {th }}$ subcarrier, and $\mathrm{E}$ denotes expectation.

From Fig.4, it is clear that as $\rho$ decreases, the average spectral efficiency also decreases. For SNR of $15 \mathrm{~dB}$, we get spectral efficiencies as $0.4,2.3,3$ corresponding to values of $\rho$ such as $0,0.5,1$.

\section{CONCLUSIONS}

The effects of feedback delay on the performance of a MISOOFDM system have been analyzed in this paper. The feedback delay degrades the overall performance of the system. Through analytical modeling and simulation results, it is clearly visible that average BER and average Spectral efficiency performance of MISO-OFDM system decreases, when correlation $(\rho)$ is less than 1 .

\section{REFERENCES}

[1] Bolcskei H. "MIMO-OFDM wireless systems: basics, perspectives and challenges". IEEE Wireless Commun; vol.13, no.4, pp.31-37, August 2006.

[2] E. Biglieri, G. Caire, and G. Taricco, "Limiting performance of block fading channels with multiple antennas," IEEE Trans. Inf. Theory,vol.47, no. 4, pp. 1273-1289, May 2001.

[3] A. J. Goldsmith and P. P. Varaiya, "Capacity of fading channels with channel side information" IEEE Trans. Inf.Theory, vol. 43, no. 6, pp.1986-1992, Nov. 1997.

[4] Trivedi YN, Chaturvedi AK. Performance analysis of multiple input single output systems using transmit beamforming and antenna selection with delayed channel state information at the transmitter. IET Commun vol.5, no.6, pp.827-34, 2011.

[5] Y.N. Trivedi "Performance analysis of OFDM system with transmit antenna selection using delayed feedback"Int. J. Electron. Commun. (AEU), vol.67, pp.671-675, 2013.

[6] Ramya TR, Bhashyam S., Sajith Rajan, "Using delayed feedback forantenna selection in MIMO systems". IEEE Trans Wireless Commun vol.8, no.12, pp.60596067, December 2009.

[7] Erik Dahlman, Stefan Parkvall, Johan Skold, Per Beming, 3G Evolution: HSPA and LTE for Mobile Broadband

[8] T.S. Rappaport, Wireless Communications, $2^{\text {nd }}$ Edition, Prentice Hall PTR, 2002.

[9] Z. Chen, J. Yuan, and B. Vucetic, "Analysis of transmit antenna selection/maximal ratio combining in Rayleig fading channels". IEEE Trans. Veh. Technol. vol.54, no.7, pp. 1312-1321, July 2005.

[10] Vinoth Babu, K., Ramachandra Reddy, G., Gupta, B. \& Sneha Reddy, D. 2013, "Improvement of quality of service (QOS) in MISO-OFDM systems using superposition based adaptive modulation (SPAM) and space frequency block coding (SFBC) technique", International Journal of Engineering and Technology, vol. 5, no. 3, pp. 2108-2115.

[11] Chen C, Sezgin A, Cioffi JM, Paulraj A. "Antenna selection in space-time block coded systems: performance analysis and low-complexity algorithm". IEEE Trans Signal Process vol.56, no.7, pp.33033314, July 2008.

[12] David HA. "Order statistics". New York: Wiley; 1970.

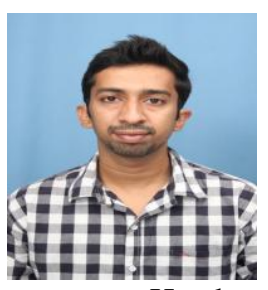

\section{BIOGRAPHIES}

Suraj Gawande is pursuing his M Tech in Communication Engineering in School of Electronics Engineering,VIT University, Vellore, Tamil Nadu, India. $\mathrm{He}$ is presently doing research on performance analysis of MIMO OFDM systems. He has completed B Tech in Electronics and Telecommmunication from RTMNU. His research interests include Wireless Communications and Signal Processing.

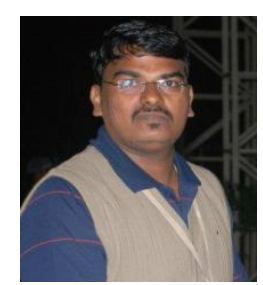

K.Vinoth Babu is presently working as an Assistant Professor (Senior) in School of Electronics Engineering, VIT University, Vellore, Tamil Nadu, India. $\mathrm{He}$ has completed his M.Tech in Communication Engineering from VIT University and B.Tech in Electronics and Communication Engineering from Anna University. He is presently doing $\mathrm{PhD}$ in VIT University. His area of interests includes Wireless Digital Communications and Signal Processing. $\mathrm{He}$ is presently working in QoS improvement in MIMO-OFDM Systems. Prof. Babu is a member in IEEE. He has conducted various workshops and Faculty Development Programs in the area of wireless communications. He has guided more than 40 projects in the field of wireless communication.

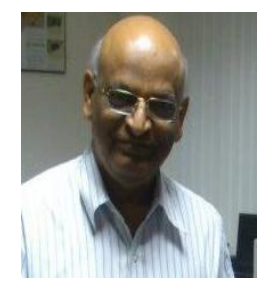

Dr.G.Ramachandra Reddy is presently working as a Senior Professor \& Dean in School of Electronics Engineering, VIT University, Vellore, Tamil Nadu, India. He has received M.Sc. and M.Sc.(Tech) degrees from Birla Institute of Technology (BITS) ,Pilani, India in 1973 and 1975 respectively and the Ph.D degree from IIT (Madras) at Chennai in 1987.His research interests include Signal Processing and Wireless Communications.Prof. Reddy is a Senior member in IEEE. He has conducted various workshops and Faculty Development Programs in the area of wireless communications and signal processing. 


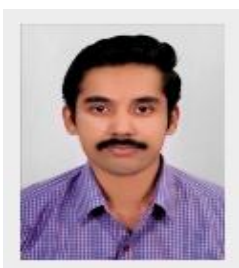

Bibin Baby John is pursuing his M Tech in Communication Engineering in School of Electronics Engineering,VIT University, Vellore, Tamil Nadu, India. He is presently doing research on improving the energy efficiency in MIMO OFDM systems using a combination of antenna selection and bit loading algorithms. He has completed B Tech in Electronics and Commmunication from Mahatma Gandhi University, Kottayam. His research interests include energy efficient data transmission and coding techniques for wireless communications systems. 